\title{
Impact of Big Data Analytics on Healthcare and Society
}

\section{Ajit Kumar Roy*}

Central Agricultural University, Agartala, Tripura, India

*Corresponding author: Ajit Kumar Roy, Central Agricultural University, Agartala, Tripura, India, Tel: 9433089634; E-mail: akroy1946@yahoo.co.in

Rec date: April 01, 2016; Acc date: April 22, 2016; Pub date: April 29, 2016

Copyright: ( 2016 Kumar RA. This is an open-access article distributed under the terms of the Creative Commons Attribution License, which permits unrestricted use, distribution, and reproduction in any medium, provided the original author and source are credited.

\begin{abstract}
Big data is created every day by the interactions of billions of people using computers, GPS devices, cell phones, censors and medical devices, data-intensive areas such as atmospheric science, genome research and, astronomical studies. Today big data opens huge opportunities to those who can use it effectively. Now realizing the great importance of big data, many analytical companies are engaged in finding hidden information in big data. According to internet experts the present technological advances to collect and analyze massive sets of data is likely to lead to revolutionary changes in business, and society. Till date a lot of work has been done on the tools, software, platforms, analytics etc. applied to big data. Many organizations are giving attention in big data analytics for development, education, disaster management, health care, and natural resource management for benefit of society. Therefore, it is attempted to compile and document the real use cases, benefits, advantages, impact and future challenges of big data. UN Global Pulse has worked on several research projects in collaboration with public and private partners demonstrating the beneficial effect of analytics from monitoring early indicators of unemployment hikes to tracking fluctuations of commodity prices before they are recorded in official statistics. According to thought leaders big data is already showing the potential in areas as genetic mapping and personalized e-commerce. The unprecedented growth in processing power and software technologies such as Hadoop, are allowing organizations "to make decisions that simply could not be made before" having profound impact. Its influence is felt in business planning, research, sales, production and elsewhere. These are considered as new industrial revolution. Scientists used to decode human DNA in minutes, find cures for cancer, accurately predict human behaviour, optimise marketing efforts, prevent diseases and foil terrorist attacks, utilising big data. Finally, concerns about privacy expressed by experts cannot be ignored. As many companies use our private information. The presentation is focused on how Big Data Analytics impact health care and Society.
\end{abstract}

Keywords: Big data; Velocity; Human-sourced information; Healthcare; Society

\section{Introduction}

Big Data: Broadly data that is difficult to collect, store or process within the conventional systems is termed as big data. Big data is used to describe data that is high volume, high velocity, and high variety. It requires new technologies and techniques to capture, store, and analyze aiming to enhance decision making, provide insight and discovery. Volume refers to the amount of data expressed in terabytes and, petabytes of data. Variety refers to the number of types of data that includes unstructured data in the form of text, video, audio, click streams, 3D data and log files. Velocity on the other hand refers to the speed of data processing data streams from mobile devices, click streams, high-frequency stock trading, and machine-to-machine processes is massive and continuously fast moving.

Big data analytics helps unearth hidden patterns, unknown correlations, and other business information using big data tools capable to analyze high-volume, high-velocity and high-variety information assets far better than conventional tools. Companies are using new big data technologies and solutions such as Hadoop, MapReduce, Hadoop Hive, Spark, Presto, Yarn, Pig, NoSQL databases and more to support their big data analytics.

\section{Sources of big data}

- Social Networks: Face book, Twitter, Tumblr etc.
- Blogs and comments

- Personal documents

- Pictures: Instagram, Flickr, Picasa etc.

- Videos: YouTube etc.

- Internet searches

- Mobile data content: text messages

- User-generated maps

- Social Media Communications

- Data from sensors: Fixed sensors; Home automation; Weather/ pollution sensors; Traffic sensors/webcam; scientific sensors; Security/surveillance videos/images

- Mobile sensors (tracking): Person (Mobile phone location); Road (Car, Trucks); Rail (Trains); Air (planes); Nautical (Ships)

- Satellite data: Topographic; Thermal; Surveillance; Meteorological; Others

- Data from computer systems: Logs; Web logs

A useful perspective is to characterize big data as having high volume, high velocity, and high variety - the three Vs [1].

- High volume - the amount or quantity of data

- High velocity - the rate at which data is created

- High variety - the different types of data

\section{$1^{\text {st }}$ Character of big data-volume}

- A typical PC might have had 10 gigabytes of storage in 2000. 
Page 2 of 7

- Today, Face book ingests 500 terabytes of new data every day.

- Boeing 737 will generate 240 terabytes of flight data during a single flight across the US.

- The smart phones, the data they create and consume; sensors embedded into everyday objects will soon result in billions of new, constantly-updated data feeds containing environmental, location, and other information, including video.

\section{$2^{\text {nd }}$ Character of big data: velocity}

- Click streams and ad impressions capture user behavior at millions of events per second.

- High-frequency stock trading algorithms reflect market changes within microseconds.

- Machine to machine processes exchange data between billions of devices.

- Infrastructure and sensors generate massive log data in real-time.

- On-line gaming systems support millions of concurrent users, each producing multiple inputs per second.

\section{3rd Character of Big Data-Variety}

Big Data isn't just numbers, dates, and strings. Big Data is also geospatial data, 3D data, audio and video, and unstructured text, including log files and social media.

- Traditional database systems were designed to address smaller volumes of structured data, fewer updates or a predictable, consistent data structure.

- Big Data analysis includes different types of data.

\section{Estimates of big data volume}

- In 2020, there will be 35 zettabytes of digital data. That represents a stack of DVD's that would reach half way from the Earth to Mars.

- Facebook has 70 petabytes and 2700 multiprocessor nodes.

- The Bing search engine has 150 petabytes and 40,000 nodes.

- The simplest response comes from Forrester Research and is as follows:

- Big Data: Techniques and Technologies that Make Handling Data at Extreme Scale Economical.

- In 2011,Hilbert and Lopez estimated that if all the data used in the world today were written to CD-ROMs and the CD-ROMs piled up in a single stack, the stack would stretch all the way from the Earth to the Moon and a quarter of the way back again.

- A report by the International Data Corporation in 2010 estimated that by the year 2020 there will be 35 Zettabytes (ZB) of digital data created per annum.

- Social media, web analytics, log files, sensors, and such like all provide valuable information, while the cost of it solutions continues to drop and computer-processing power is increasing.

- The Internet of Things is also becoming an increasingly rich source of data. At this moment, there are 13 billion devices connected to the Internet and that will be 50 billion in 2020 says Cisco CTO Padmasree Warrior.

- IDC expects more than 1 billion sensors to be connected to the Internet by that time. All the accompanying data flows can supply interesting insights that can aid better business decisions.
- Social media, web analytics, log files, sensors, and such like all provide valuable information, while the cost of it solutions continues to drop and computer-processing power is increasing.

- The Internet of Things is also becoming an increasingly rich source of data. At this moment, there are 13 billion devices connected to the Internet and that will be 50 billion in 2020 says Cisco CTO Padmasree Warrior.

\section{What is big data analytics?}

The definition of big data holds the key to understanding big data analytics. According to the Gartner IT Glossary, Big Data is highvolume, high-velocity and high-variety information assets that demand cost effective, innovative forms of information processing for enhanced insight and decision making.

\section{Tools used in big data analytics}

- Where processing is hosted? Distributed Servers/Cloud (e.g. Amazon EC2)

- Where data is stored? Distributed Storage (e.g. Amazon S3)

- What is the programming model? Distributed Processing (e.g. MapReduce)

- How data is stored and indexed? High-performance schema-free databases (e.g. MongoDB)

- What operations are performed on data? Analytic/Semantic Processing

\section{Techniques and Methods of Analytics}

\section{Learning analytics draws upon techniques from a number of established fields}

Statistics; Artificial Intelligence;Machine Learning; Data mining; Social Network Analysis; Text Mining and Web Analytics; Operational Research; Testing; Cluster Analysis; Classification; Network analysis; Predictive modelling; Sentiment analysis; Information Visualization.

\section{Big data Usage}

E-Commerce and market intelligence: recommender system;-Social media monitoring and analysis;-Crowd-sourcing systems;-Social and virtual games

E-Government and politics: Ubiquitous government services; Equal access and public services; Citizen Engagement

Science and Technology: S\&T innovation; Hypothesis testing; Knowledge discovery

Smart Health and Wellbeing: Human and plant genomics; Healthcare decision support; Patient community analysis

Security and Public Safety: Crime analysis; Computational criminology; Terrorism informatics; Open-source intelligence; Cyber security.

\section{The benefits of big data analytics}

Business value of big data is accrued when the data is analyzed properly not just collecting and storing. The benefits of using analytics in decision making to the extent of 5-6\% higher productivity compared to the conventional business firms according to IBM Institute for 
Page 3 of 7

Business Value that surveyed a global sample of nearly 3,000 executives $[2,3]$.

\section{Surprising statistics about big data}

It is reported that seven out of $10 \mathrm{CIOs}$ and other top executives say data analytics is a "crucial" or "very important" business driver, according to survey research from KPMG. Digital universe is doubling every two years, and will reach 40,000 Exabyte (40 trillion gigabytes) by $2020[4]$.

\section{Big data: interesting facts and figures}

It is well known that it took from the dawn of civilization to the year 2003 for the world to generate 1.8 zettabytes of data. In 2011 it took two days on average to generate the same amount of data. Data centers consume up to 1.5 percent of all the electricity in the world [5].

\section{Opportunities to Generate Knowledge from Big Data}

Despite the obstacles and the risks, the potential value of Big Data is inestimable. A hint at future gains from Big Data comes from the National Science Foundation's (NSF) 2012 solicitation for grants in core techniques for Big Data (BIGDATA NSF12499).

\section{The NSF envisions a big data future with the following pay- offs:}

- Responses to disaster recovery for effective.

- Enable biomedical discovery and patient-centered therapy utilizing health/disease/genome/environmental knowledge bases.

- Management of increasingly stressed watersheds and eco-systems through development of forecasting models.

- Access to data and software available to everyone.

- Optimal energy consumption decisions based on information the consumers homes and cars.

- Identify at-risk man-made structures like bridges, moderate the impact of failures, and avoid disaster;

- Students and researchers have intuitive real-time tools to view, understand, and learn from publicly available large scientific data sets on genome sequences to astronomical star surveys, and public health databases.

- Accurate predictions of earthquakes, hurricanes, and tornadoes, enable life-saving and cost-saving preventative actions.

- Many of these hopes for the future may come true if we manage our Big Data resources wisely.

\section{Five ways to leverage big data}

1. Big Data can unlock significant value by making information transparent.

2. As organizations create and store more transactional data in digital form

3. Big Data allows ever-narrower segmentation of customers and therefore much more precisely tailored products or services.

4. Sophisticated analytics can substantially improve decisionmaking, minimize risks, and unearth valuable insights that would otherwise remain hidden.
5. Big Data can be used to develop the next generation of products and services.

\section{Business-benefits-from-big-data}

The power of insights provided by big data created by the timely, meaningful outcome from large data sets is often helped in the effective enterprise decision-making maximizing business impact. Altogether people, process and analytic tools are required to have some of the potential business benefits.

\section{Impact of Big Data on Healthcare}

\section{Ways big data can change healthcare}

Perhaps fiscal concerns, more than any other factor, are driving the demand for big-data applications. After 20 years of steady increases, health-care expenses now represent 17.6 percent of GDP nearly $\$ 600$ billion more than the expected benchmark for a nation of the United States' size and wealth [6,7]. The report outlines five ways data will enable the healthcare industry to cut costs and improve quality.

Right living: Data can help patients to take an active role in their own health such as diet, exercise, and medication adherence to take control of their health.

Right care: Data can improve outcomes, reducing medical errors. Application of big data tools will facilitate evidence-based care that is personalized to the specific patient.

Right provider: Proven outcomes for patients to receive the best medical care based on data that helps us better match the provider's skill set with the needs of the patient and allow assessment of specific providers.

Right value: Cost-effective healthcare through different methods, such as patient-outcome reimbursement and eliminating fraud, waste, and abuse in the system utilizing big data

Right innovation: Innovators will be able to address all aspects of therapeutic innovation discovery, development, and safety utilizing data from past trials as well as analyzing trends from current data. Healthcare providers can analyze patient history data, real-time data from monitors, clinical factors, lifestyle choices and social determinants to provide a holistic view of the patient and develop the most effective care plans. IBM has helped healthcare providers:

Identify crises before they happen and treat patients proactively by analysing data in real time as it streams from monitoring equipment.

Predict patient health risks using predictive analytics to understand underlying clinical or social factors, and design more effective care plans.

Improve healthcare outcomes by providing timely and meaningful insights to care providers, who can then administer the most effective treatments.

\section{The Big Players in Big Data}

There is a good deal of buzz around Big Data-hardly a week goes by without a new start-up or business preaching the virtues of mining massive tracts of information. Understandably, many have trouble seeing beyond the hype, but nowhere are the benefits of Big Data more tangible than in the healthcare sector. 
Page 4 of 7

- IBM predicts a $20 \%$ decrease in patient mortality as the medical field gears up to analyse streaming patient data with large-scale software applications. That's not just a return on investment though it is, that's using information to save human lives. Even now, major corporations like Microsoft, Dell, IBM, and Oracle are pioneering data-mining platforms that will help medical professionals stay on top of patient data and provide improved medical care.

- The Big Data industry deals primarily in collections of data that are beyond the ability of common software to capture, process, and manage in a timely manner. The amount of data processed can range from a few dozen terabytes to many petabytes of information. A plethora of Big Data tools have been released recently to make meaning of it all you could say they're finding new ways to make data earn its space. Read on to learn more about Big Data's colossal impact on the healthcare industry.

- Mining Data to Save Lives: The biggest risk any patient takes when admitted to a hospital is being examined by another human. Missed warning signs, overlooked risk factors, and cursory assessments are often part and parcel of hectic admission wards. That's why healthcare providers are beginning to turn to computers to help them make quick and accurate decisions about patient health. There are endless exciting opportunities for Big Data in the future of healthcare:

- Reducing Readmissions. What if a pile of data could tell you how likely a patient was to be readmitted after treatment? Doctors would able to judge whether patients would benefit from short or longer stays, as well as track specific treatment factors leading to the reoccurrence of ailments. That saves time (and money) for both the doctor and the patient.

- Accessing Data Anywhere. Using secure data querying technologies capable of parsing enormous amounts of information, it's possible for medical professionals to access remote data for a more timely and completes understanding of their patient's ailment.

- Point-Of-Care Decision-Making. Imagine tools and equipment with built-in data processing capable of helping doctors make instant, life saving calls at point-of-care. The most obvious application for this is in fast-paced areas such as the hospital emergency room.

- Innovative Smart Devices. We're already surrounded by intelligent devices capable of funnelling large amounts of data at light speed. Why not put a chip in an in-home diabetes monitor that can send back frequent and useful data about a patient's in-home treatment? Take it a step further, and make smart toothbrushes, smart toilets, and smart scales capable of reporting instantly on a patient's condition.

- Genome Sequencing. Though still a distant dream, whole genome sequencing may be the most intriguing item on this list. When the human genome was first decoded, it took over ten years to process the data (which is in petabytes) - now it takes merely a week. As Big Data technology expands to process even larger amounts of data, every-day genome sequencing may become available to the private sector.

- Many of these possibilities are already in the process of being implemented, but some possibilities are a little farther off. Either way, it's encouraging to consider that the amassment of huge quantities of data-a practice frowned upon by many privacyloving citizens can and will have a profound effect on lives saved and improved by medical technology.

\section{Big data analytics for well-being: tracking infectious disease}

Department of Health in Hong Kong modernized its analytics to link many different systems for a better flow of information after the SARS outbreak of 2003. The department can identify hotspots to forecast where disease is likely to spread next. The department is now better prepared to fight the next health emergencies, including a more recent outbreak of Dengue fever. Similar analytics approaches are now being used around the world [8]

Analytics helped aid workers prioritize assistance levels and supply distribution after Typhoon Haiyan devastated the Philippines in 2013, big data analytics made relief efforts more accurate and responsive, which in turn made the country more resilient in recovering from the disaster, reduced suffering, and saved numerous lives.

The Clinton Health Access Initiative (CHAI) uses analytics to create updated forecasts of demand for medications for HIV/AIDS, malaria, and tuberculosis, which has led to greater availability and the ability to negotiate lower prices on the drugs. CHAI also analyzes global HIV treatment cost drivers, shares forecasts and models with the United Nations Programme on AIDS and the World Health Organization, and develops treatment models in partnership with health ministries around the globe to identify how best to spend limited resources to save more than 90 percent of the 33 million people living with HIV/ AIDS reside in developing nations with limited access to treatment

Grameen Foundation's Community Knowledge Worker program in Uganda to identify disease outbreaks. The mobile space you have fantastic organizations such as MedicMobile, FrontlineSMS, ZipDial, and PharmaSecure.

Big Data can also have a negative social impact if leveraged improperly. Information can be used by bad actors to exploit those vulnerable populations. Especially when working with personally identifiable information such as names, health information, phone numbers, addresses, or geo locations.Cre may be taken the institutions protect the privacy and civil liberties of those that they are seeking to help.

Developed countries are responding to the benefit of big data, and using it as a tool to solve their long-standing problems. Big data strategies for the healthcare and social service sectors were formulated based on an ICT-based policy [9].

\section{How big data impacts healthcare}

- Google Flu Trends: a service that predicts and locates outbreaks of the flu by making use of information aggregate search queries.

- The San Francisco-based Global Viral Forecasting Initiative (GVFI) uses advanced data analysis on information mined from the Internet to identify comprehensively the locations, sources and drivers of local outbreaks before they become global epidemics

- Analysis of mobile and Internet data could lead to huge public gains and social welfare.

- Health has been the first area of commercial application of this technology for IBM.Watson's ability to understand questions and context, and to rifle through 200 million pages of data and provide precise responses in just seconds, can help a physician treating a patient to consider all related texts, reference materials, prior cases, and the latest knowledge in journals and medical literature.

- Accenture targets the $4 \%$ chronically ill patients that tie up more than $60 \%$ of the hospital resources Through a service that monitors 
Page 5 of 7

the chronically ill patients that can live at home, avoiding readmission to hospital.

- Monitoring patients' conditions is made on the basis of medical and lab records, as well as self-reporting of key parameters from instruments available to the patients.

- In the area of patient monitoring and management, many hospitals have started to apply big data analytics in the care of chronically ill patients to reduce cost per patient and to avoid unnecessary admissions to hospitals.

- IBM, through their 'Smarter Cities' initiatives, has support home care of chronically ill patients (e.g. Stavanger Hospital in Norway) $[10]$.

\section{Impact on Life Science}

\section{Big data can also be seen in the life sciences where big sets of data such as}

- Analytics on Genome sequencing and clinical data and patient data can advance breakthroughs in science in research.

- Human Genome Project is determining the sequences of the three billion chemical base pairs that make up human DNA aiming innovative biomedicine.

- Analytics is leading standard medical practice from relatively adhoc and subjective decision making to evidence-based healthcare.

- Healthcare Data is becoming more complex

- In 2012, worldwide digital healthcare data was estimated to be equal to 500 petabytes

\section{Big data is developing most rapidly in the world of big science}

- In future 2800 radio telescopes in the Square Kilometer Area project (ska), the largest Big Science project ever, will generate 1 billion gigabytes of data daily.

- The Large Hadron Collider, at the European Organisation for Nuclear Research (CERN), which has 150 million sensors and is creating 22 petabytes of data in 2012 .

- The Earth scope is the world's largest science project. Designed to track North America's geological evolution, this observatory records data over 3.8 million square miles, amassing 67 terabytes of data. It analyzes seismic slips in the San Andreas fault, sure, but also the plume of magma underneath Yellowstone and much, much more [11].

- The volume of data available to us is so large, that it is both presenting many new opportunities for analysis as well as requiring new modes of thinking [12].

- Analytics is required on data available from the atmosphere and the land, ocean and ice surfaces of planet Earth with pixel sizes ranging from $50 \mathrm{~cm}$ to many tens of kilometres from over 200 satellites in orbit continuously collecting data.

\section{Impact on business, trade and commerce}

Opportunities: It is fact that projects should address a business need, such as solving a problem or capturing an opportunity. Media attention given to big data has created an awareness of its potential, leading some enterprises to give attention to big data projects without clearly defined goals. Opportunities for big data analytics in different industries are as follows [13].
- Automobile insurance

- Telecommunications

- Manufacturing, distribution, and retail

- Transportation and logistics

- Utilities like smart meters to forecast energy demand.

- Gaming

- Law enforcement

\section{Big data is also transforming the retail market}

- Wal-Mart's transactional databases ?more than 2.5 petabytes of data consisting of customer behaviors and preferences, network and device activity, and market trends data.

- Wal-Mart's inventory management system enables suppliers to see the exact number of their products on every shelf of every store at each precise moment in time.

- Amazon's "Customers Who Bought This Also Bought" features, prompting users to consider buying additional items. Amazon has evolved into a complete retail platform/store.

- One of Amazon's key success factors was the early use/adaption of machine learning and big data for targeted marketing, with as much as $30 \%$ of the sales driven by automatic customer recommendations.

- Major use of BI and loyalty program Outsourced to Dunnhumby for analysis. $80 \%$ of customers are Clubcard members Constantly updating data - every transaction improves data quality

- Targeted promotions both direct and sponsored by suppliers (revenue

stream)

- But Tesco is a financial services company as well Founded in 1995,

- eBay Inc. is an American multinational consumer-to-consumer Internet corporation with presence in over thirty countries. EBay is now a multibillion dollar business with a business model of simply providing a network that connects buyers and sellers of goods and where anyone can trade.

\section{Value Chain Optimization}

Big data capabilities enable companies such as

- FedEx,

- Wallenius Wilhelmsen (car carrier ship owner,),

- Maersk "Daily Maersk conveyor belt" (container ship owner),Guaranteeing daily shipments between major Asian and European ports

- Accenture developed an Asset Analytics solution for Canadian utility Hydro One, based on Hydro One's existing ERP, GIS, data historian, and other legacy asset systems. It helps them with improving CAPEX/OPEX investment decisions, provides a common understanding of asset risk, supports regulatory compliance, and facilitates communications.

- OstiaEdge ${ }^{\oplus}$ enables improved use of onboard data to help implement a Condition-Based Maintenance (CBM strategy).This creates value for the operators, managers, and owners in a variety of ways; Prioritizes maintenance spend across a fleet of assets to maximise return on investment.

- Lloyd's List Intelligence is founded on the heritage of Lloyd's List plus a global network of specialist sources of business-critical maritime data. It brings together the expertise of a global staff of 
Page 6 of 7

maritime analysts and journalists with an extensive system of shore based and satellite intelligence gathering to create a complete information support service for the maritime industry.

- Real-time analysis helps make contingency plans for different situations - for example, a sudden rise in oil prices, or a closure of a major port. The solution enables planning for best- and worstcase scenarios as well as the standard forecast, enabling the company to respond more effectively to normal demand fluctuations as well as emerging situations, such as the 2011 Japanese tsunami.

- Wallenius Wilhelmsen Logistics is saving in the order of 100 million dollars annually from route planning and general reductions in speed.

- Carfax is a US company and system that allows car owners to collect and store the complete history of a car in a central database. Whenever the car visits a workshop, the work done and the condition of the car is uploaded to the system. For the car owner, being part of network increases the second-hand value of the car. The system has added extra value.

- Another story, on how the use of data literally changed the game, is told in the book and movie. "Moneyball" is the story about how available data and analytics were applied in a new way that challenged, and entirely altered, the traditional way of valuing baseball players. This has completely transformed the way in which players in the US National baseball league are valued, and similar methods are also being applied in other sports.

- Enormous amount of stock exchange, banking, online and onsite purchasing data flows through computerized systems every day. These are captured and stored for inventory monitoring, customer behaviour and market behaviour [14].

\section{Use of big data in finance}

- Insurance uses

- Fraud Detection and Analysis

- Personalised Pricing

- Customer Sentiment Analysis

- Catastrophic Planning

- Call Detail Record

- Loyalty Management

- Social Media Analytics

- Advertising and Campaign Management

\section{Impact of big data on business, economy and society}

Big data is already showing potential in many areas "to make decisions that simply could not be made before, to handle all sorts of data questions."And that will have resounding impact. "We see this as just as profound an inflexion point as the first machine age. Big data will have an impact on all industries and every process. Its influence will be felt in business planning, research, sales, production and elsewhere. In Kawatsuma's view, this amounts to nothing less than new industrialrevolution.

\section{Big data analytics to stop leakage benefiting for social programs}

Fighting fraud that much-needed funds are available for social welfare ensuring well-being of people. Belgium's VAT losses had been reduced by 98 percent by 2012 and continue to use hybrid detection techniques to save billions of euros. This money can now be put to good use, for inclusive growth [15].

\section{Impact on energy sector}

- Grid analytics: Outage management; Voltage optimisation; Asset Management

- Consumer analytics: Revenue protection; Load forecasting Detailed customer segmentation Energy efficiency, advice and assessment

- WIND Industry: There are already many untapped sources for insights and performance enhancements in the data available in the wind industry today.

- Offshore oil and gas: In the oil and gas industries, companies have grappled with big data challenges for quite some time, for example to process seismic data. The new trend is that data and analytics are playing larger roles in other parts of the operations such as integration of offshore operations across offshore assets and system barriers, improving efficiency of drilling operations and optimizing production processes etc.These uses of data pave the way for further improving reliability and managing risk across the whole enterprise, including the risk of environmental impact as exploration and production move into more sensitive areas.

- In order to help improve productivity at its Arctic facilities, ConocoPhillips wanted to monitor and forecast ice floe movement in or near real-time. Big data technologies helped the company to collect and manage thousands of data points per second from multiple sources. Through predictive analytics, ConocoPhillips can visualize in real-time the position of ice floe near its Arctic facilities.

\section{Big data for global development}

A new initiative by the United Nations, Global Pulse will conduct sentiment analysis of messages in social networks and text messages to help predict job losses, spending reductions or disease outbreaks in a given region. Early-warning signals to guide assistance programs in advance to, to prevent a region from poverty. The project is being run as a cooperative endeavour with five main objectives [16]

1. A Global Snapshot of Well-being through Mobile Phones,

2. Real-Time E-Pricing of Bread,

3. Tracking the Food Crisis via Online News,

4. Unemployment through the Lens of Social Media,

5. Twitter and Perceptions of Crisis-Related Stress.

The more organisations that implement a Big Data strategy, the bigger the impact on the economy will be. As the McKinsey report famously states:

- Big Data can add \$ 300 billion potential annual value to US health care,

- add $€ 250$ billion potential annual value to Europe's Public sector administration

- Create a $\$ 600$ billion potential annual consumer surplus.

- Gartner predicts that Big Data will drive up to \$232 billion IT spending through 2016.

- These are massive gains and investments that of course only occur when all organisations and governments fully start to use Big Data. 
- It does show however the impact of Big Data on the global economy, also when only $12 \%$ have started implementing a Big Data strategy.

Big data and privacy concerns: The growing globalization of data flows via big data increases the risk that people can lose control of their own data. The question remains how much the Big Data future is influenced by the Snowden revelations which have put data protection and privacy at the center of our attention.

Opportunity: access to jobs: New jobs are being created in this time of economic transition. United States is projected to have 190,000 unfilled analytics positions and a shortage of 1.5 million managers and analysts skilled in big data by 2018 . According to Gartner, by 2015 , more than 85 percent of Fortune 500 organizations will be unable to effectively exploit big data [17].

\section{The Future of Big Data}

Many see promise in the future of data analysis, some fear that work with vast stores of information could lead to privacy abuses and mistaken forecasts. The growing technological ability to collect and analyze massive sets of information, could lead to revolutionary changes in human society. Leading technologists and researchers around the world look forward to the positive impact of Big Data; many also have reservations about potential benefits [17-19].

\section{Acknowledgements}

I am thankful for giving me an opportunity to deliver Key Note Address on 'Impact of Big Data Analytics on Business, Economy, Health Care and Society' at the 4th International Conference and Exhibition on Biometrics and Biostatistics conducted by OMICS International Conferences held during November 16-18, 2015, San Antonio, USA; Venue: Hilton San Antonio Airport, San Antonio, USA.

\section{References}

1. Russom P (2011) Big Data Analytics. TDWI Best Practices Report. Fourth Quarter.
2. Brynjolfsson E, Hitt L, Kim H (2011) Strength in Numbers: How does data-driven decision-making affect firm performance? Social Science Research Network.

3. Davenport TH, Harris JG, Morison R (2010) Analytics at Work: Smarter Decisions, Better Results. Boston: Harvard Business School Press, USA.

4. http://www.baselinemag.com/analytics-big-data/slideshows/surprisingstatistics-about-big-data.html\#sthash.K0mcOHk2.dpuf

5. http://www.chassis-plans.com/blog/big-data-interesting-facts-andfigures

6. http://www.utopiainc.com/insights/blog/381-7-biggest-businessbenefits-from-big-data

7. http://www.mckinsey.com/industries/healthcare-systems-and-services/ our-insights/the-big-data-revolution-in-us-health-care

8. Hagström M (2014) From-Sars-To-Ebola-How-Big-Data-Fights-Disease. World Economic Forum.

9. http://www.ncbi.nlm.nih.gov/pubmed/25705552

10. https://www.whitehouse.gov/share/brain-initiative

11. http://www.nbcnews.com/id/44363598/ns/technology_and_sciencefuture_of_technology/\#.TmetOdQ--uI

12. http://virtualobservatory.org

13. Franks B (2012) Taming The Big Data Tidal Wave: Finding Opportunities in Huge Data Streams with Advanced Analytics. Wiley.

14. Roy A (2015) Impact of Big Data Analytics on Business, Economy, Health Care and Society. Paradox,Future: Big Data Analytics for Social Welfare. Kindle Edition.

15. http://www.ap-institute.com

16. http://www.unglobalpulse.org/sites/default/files/ BigDataforDevelopment-UNGlobalPulseJune2012.pdf

17. Gartner (2012) Gartner Says Big Data Creates Big Jobs: 4.4 Million IT Jobs Globally to Support Big Data By 2015, Gartner Press Release, USA.

18. Watson HJ, Marjanovic O (2013) Big Data: The Fourth Data Management Generation, Business Intelligence Journal 18: 4-7.

19. Manyika (2011) Big Data: The Next Frontier of Innovation, Competition, and Productivity, McKinsey Global Institute, USA. 